\title{
Biomimetic synthesis of chiral erbium-doped silver/peptide/silica core- shell nanoparticles (ESPN)
}

\author{
Alexandre Mantion, ${ }^{\mathrm{a}, *}$ Philipp Graf, ${ }^{\mathrm{b}}$ Ileana Florea, ${ }^{\mathrm{c}}$ Andrea Haase, ${ }^{\mathrm{d}}$ Andreas F. Thünemann, ${ }^{\mathrm{a}}$ Admir \\ Mašić, ${ }^{\mathrm{e}}$ Ovidiu Ersen, ${ }^{\mathrm{c}}$ Pierre Rabu, ${ }^{\mathrm{c}}$ Wolfgang Meier, ${ }^{\mathrm{b}}$ Andreas Luch, ${ }^{\mathrm{d}}$ and Andreas Taubert ${ }^{\mathrm{e}, \mathrm{f}, *}$ \\ ${ }_{5}$ Received (in $\left.X X X, X X X\right)$ Xth $X X X X X X X X X 20 X X$, Accepted Xth $X X X X X X X X X 20 X X$ \\ DOI: $10.1039 / b 000000 x$
}

a BAM Federal Institute for Materials Research and Testing, Richard-Willstaetter-Str. 11, 12489 Berlin (Germany), alexandre.mantion@bam.de b University of Basel, Department of Chemistry, Klingelbergstrasse 80, CH-4056 Basel (Switzerland)

c Institut de Physique et Chimie des Matériaux de Strasbourg, UMR7504 CNRS-Université de Strasbourg, 67034 Strasbourg (France)

10 d BFR - Federal Institute for Risk Assessment, Department of Product Safety, Thielallee 88-92, 14195 Berlin (Germany)

e Max Planck Institute of Colloids and Interfaces, 14476 Golm (Germany)

f University of Potsdam, Institute of Chemistry,Karl-Liebknecht-Str. 24-25, 14476 Golm, (Germany) ataubert@uni-potsdam.de

Electronic supplementary information available: Figures S1 to S12, Tables S1 and S2

15 Peptide-modified silver nanoparticles have been coated with an erbium-doped silica layer using a method inspired by silica biomineralization. Electron microscopy and small-angle X-ray scattering confirm the presence of an Ag/peptide core and silica shell. The erbium is present as small $\mathrm{Er}_{2} \mathrm{O}_{3}$ particles in and on the silica shell. Raman, IR, UV-Vis, and circular dichroism spectroscopies show that the peptide is still present after shell formation and the nanoparticles conserve a chiral plasmon resonance. Magnetic

20 measurements find a paramagnetic behavior. Cultivation tests show that the resulting multicomponent nanoparticles have a low toxicity for macrophages, even on partial dissolution of the silica shell.

\section{Introduction}

Silver nanoparticles are important building blocks for the creation of new materials with tailored properties for optical, ${ }^{1}$ 25 sensing, ${ }^{2}$ and medical applications; ${ }^{3-5}$ many synthesis protocols therefore exist. ${ }^{6-8}$ Current efforts focus on tuning of particle properties including particle-particle, particle-surface, or particlebiology interactions; preservation or induction of chirality; coupling of optical to biological signals, etc. ${ }^{9-11}$ Short peptides 30 can efficiently control the shape, size, and organization of silver nanoparticles ${ }^{12-16}$ and chiral induction from the peptide to the metallic structure is possible. ${ }^{17,18}$ At the same time, peptides considerably improve the colloidal and chemical stability ${ }^{12,} 19$ leading to multifunctional and responsive nanoparticles.

35 Silver nanoparticles are, however, quite reactive and must be protected from etching, leaching, oxidation, and coagulation. This is typically done via coating with thiols or an inert inorganic coating like silica. ${ }^{20}$ Silica has many advantages as the chemistry (and the resulting structures), surface modification, and properties 40 (colloidal, ${ }^{21}$ optical, ${ }^{22-24}$ etc.) can be accurately controlled. To date, however, the synthesis of a well-defined, dense but thin (a few $\mathrm{nm}$ ) silica layer on individual nanoparticles has been difficult. Instead, ill-defined and aggregated structures form and only few protocols enable a controlled silica deposition. ${ }^{20,25-34}$

45 We have recently developed a peptide-based, biomimetic process for coating silver nanoparticles with a uniform silica layer of 1 to $4 \mathrm{~nm} .{ }^{35}$ Among others, this coating is interesting because it can act as a host for ions and molecules leading to an even more pronounced (multi)functionality of the hybrid 50 nanoparticles. Among others, dyes or metals (e.g lanthanides) can be incorporated into the silica layer, leading to a wealth of possible applications in, e.g., diagnostics or imaging. ${ }^{36-40}$

Erbium has attracted attention for its near-infrared luminescence. The characteristic emission at $1.53 \mu \mathrm{m}$ is relevant 55 for telecommunication, as it is located in a region where the absorption of glass optical fibers is minimal. ${ }^{41}$ Unfortunately, erbium ions are poorly sensitive and have a low fluorescence efficiency. Therefore, ytterbium, ${ }^{42,} 43$ silicon, ${ }^{44,} 45$ and silver nanoparticles ${ }^{46,47}$ have been used as sensitizers, alone or in 60 combination. Hybrid materials based on glass, erbium, and silver nanoparticles have also been studied. ${ }^{43}$, $48-50$ This work has been complemented by theoretical efforts to understand the physics of the energy transfer between silver nanoparticles and erbium, ${ }^{51-53}$ similar to other lanthanides. ${ }^{54}$ Finally, complexation with organic 65 ligands and incorporation into xerogels $5^{55,56}$ or biological material ${ }^{57-59}$ has also been explored.

In spite of the many useful applications, there are no reports on biomimetic, peptide-based lanthanide/silica/silver hybrids. The current manuscript is thus the first report on complex, 70 multifunctional erbium-doped silver/peptide/silica nanoparticles (ESPN) obtained by a soft biomimetic process. The hybrid particles have interesting properties such as chiral induction of the metal core, well-defined and biocompatible shell, chiral information encoded in the peptide/silica shell, and characteristic 75 optical and magnetic properties. 


\section{Experimental section}

General. Chemicals were obtained from Bachem (Bubendorf, Switzerland), Fluka (Buchs, Switzerland), or ABCR (Karlsruhe, Germany) and used as received. Amino-acids are L-amino acids.

5 Silver nanoparticles. Peptide 1 (Scheme 1) and silver nanoparticles were prepared as published ${ }^{12}$ and directly used after preparation. Particles were purified by repeated centrifugation to remove free, unbound peptide. The particles are around $20 \pm 4$ $\mathrm{nm}$ in diameter and almost exclusively coated by the covalently 10 attached peptide 1 as shown by XPS spectroscopy. ${ }^{12}$

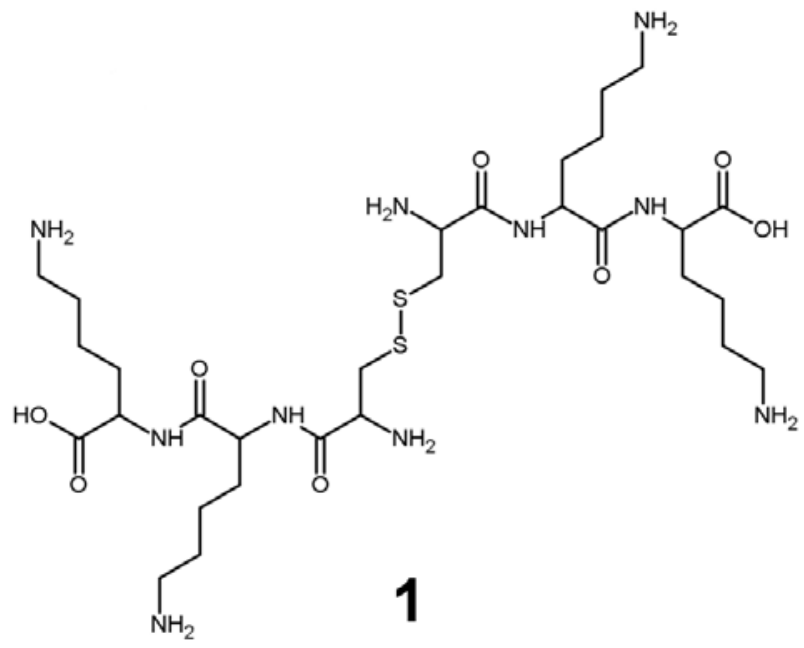

Scheme 1. Peptide 1 used for surface modification of the silver nanoparticles. The neutral form of the peptide is shown. The peptide sequence is CKK. The two sub-sequences are connected through a disulfide bridge (-S-S-). All amino acids are L-amino acids.

Silicification. To a $1 \mathrm{mg} / \mathrm{mL}$ peptide-coated silver nanoparticle dispersion ( $20 \mathrm{~mL}, \mathrm{pH} \mathrm{3}$, ice cooled) $200 \mu \mathrm{L}$ of icecooled tetraethoxysilane (TEOS) were added under strong stirring in a Teflon flask. After 2 days at $25^{\circ} \mathrm{C}$ under vigorous stirring, 20 the sample was isolated and purified by repeated centrifugation/dispersion in water. The particles were redispersed in $20 \mathrm{~mL}$ of water and $200 \mu \mathrm{L}$ of 3-aminopropyltriethoxysilane added. After 24 hrs, the particles were isolated and purified by repeated centrifugation/dispersion in water. Synthesis of the 25 erbium-doped samples was as above, but 200 or $140 \mu \mathrm{L}$ of icecooled TEOS and 3 or $30 \mathrm{mg}$ of erbium (III) triisopropoxide, respectively were added.

High resolution scanning electron microscopy. SEM images were acquired on a Hitachi S-4800 with field emission gun 30 operated at $5 \mathrm{kV}$ without sample sputtering. Substrates were glass cover slips coated with platinum (4 nm) in a BalTec MED 020. For EDX, concentrated suspensions were deposited on aluminium or carbon supports and large aggregates were analysed.

Transmission electron microscopy. TEM images were taken 35 on an FEI Morgani 268D operated at $80 \mathrm{kV}$. Samples were deposited on carbon-coated copper grids and directly imaged after drying in air. Some samples were diluted prior to imaging for better imaging conditions. High resolution TEM was done on a JEOL 2100F (FEG) TEM/STEM operated at $200 \mathrm{kV}$ with a 40 spherical aberration $\left(\mathrm{C}_{\mathrm{s}}\right.$ ) probe corrector and a Gatan TRIDIEM post-column imaging filter. Images were acquired on a 2048x2048 pixel, cooled CCD detector. STEM-ADF images were acquired using an annular detector and a camera length of 8 $\mathrm{cm}$, which corresponds to an inner semi-angle of about $40 \mathrm{mrad}$.

${ }_{45}$ Small-Angle X-Ray scattering. SAXS measurements were done on a SAXSess camera (Anton Paar, Austria) attached to a laboratory X-ray generator (PW3830, PANanalytical) with a fine focus glass X-ray tube $(40 \mathrm{kV}, 50 \mathrm{~mA}, \mathrm{CuK \alpha}, \lambda=0.1542 \mathrm{~nm})$. Samples were filled in a reusable vacuum-tight $1 \mathrm{~mm}$ quartz 50 capillary to attain the same scattering volume and background contribution. The scattering vector is defined in terms of the scattering angle $\theta$, and the wavelength of the radiation is $q=4 \pi / \lambda$ $\sin (\theta)$. SAXS data were recorded as $700 \times 1.2 \mathrm{~s}$ repetitions in a $q$ range of $0.04 \mathrm{~nm}^{-1}$ to $5.0 \mathrm{~nm}^{-1}$ with a CCD detection system 55 (Anton Paar). The two-dimensional intensity data were converted to one-dimensional data and deconvoluted using SAXSQuant (Anton Paar). Data were fitted with Igor Pro 6.0.4 (Wavemetrics) and the SANS Data Analysis Package ${ }^{60}$ (NIST) using a model where the shell thickness is constant and the core radius 60 polydisperse $^{61}$ assuming a Schultz distribution ${ }^{62,63}$ of the radii. Pair distribution functions were determined assuming a spherical symmetry using GIFT. ${ }^{64}$ DECON $^{65}$ was used to determine the radial electron density distribution $\rho(r)$. IPG-TNNLS ${ }^{66}$ (Internal Point Gradient-Total Non-Negative Least Square) analysis was ${ }_{65}$ performed as implemented in the Irena Package ${ }^{67}$ v2.38 using Igor Pro 6.21 (Wavemetrics). Data were fitted for a nanoparticle population with sizes from $3 \mathrm{~nm}$ to $100 \mathrm{~nm}$ and a logarithmic binning. The NNLS approach parameter was set to 0.5 and the maximum number of iterations was set to 300 , which was 70 sufficient for convergence.

Synchrotron X-Ray diffraction. Samples were measured in 1 mm Mark tubes at the $\mu$ Spot beamline ${ }^{68}$ at BESSY-II, using a wavelength of $1.5406 \AA$ for the ESPN and $1.0000 \AA$ for the pure nanoparticles. Silicon was used as external standard and the 2D 75 data were converted using Fit2D. ${ }^{69}$ Powder patterns were calculated using Fullprof $4.60 .^{70}$

Thermogravimetric analysis. TGA was done on a Mettler Toledo TGA/SDTA 851 e from 25 to $800^{\circ} \mathrm{C}$ at $10^{\circ} \mathrm{C}^{*} \mathrm{~min}^{-1}$ in $\mathrm{N}_{2}$.

80 Infrared Spectroscopy. IR spectra were obtained from the neat samples on a Shimadzu FTIR 8300 with a Golden Gate ATR unit from 300 to $4500 \mathrm{~cm}^{-1}$ (resolution of $1 \mathrm{~cm}^{-1}, 128 \mathrm{scans}$ ).

UV-Vis spectroscopy. Samples were measured in $1 \mathrm{~cm}$ quartz cuvettes on a Perkin Elmer Lambda 25. Data were deconvoluted 85 using Fytik as published previously. ${ }^{12}$

Surface-enhanced Raman spectroscopy (SERS). Silver nanoparticles were investigated as dispersion in water with a confocal Raman microscope (CRM300, WITec, Germany) with a piezo-scanner (P-500, Physik Instrumente, Germany), a 60x 90 objective (numerical aperture: 1), and a $532 \mathrm{~nm} \mathrm{Nd:YAG} \mathrm{laser.}$ Spectra were acquired with an air-cooled CCD detector (DU401BV, Andor, UK) with 600 lines/mm (UHTS 300, WITec, Germany). ScanCtrlSpectroscopyPlus (v2.04, WITec) was used for data acquisition and processing. Power was adjusted for good 95 signal-to-noise ratio and to avoid sample destruction. Typically, below $1 \mathrm{~mW}$ full beam power at the sample was applied. 100 spectra of $1 \mathrm{~s}$ were acquired for good signal/noise ratio.

CD Spectroscopy. CD spectra were recorded on a Chirascan CD spectrophotometer (Applied Physics, UK). Samples were 
dispersed in aqueous $\mathrm{HCl}$ at $\mathrm{pH} 3$ and experiments were done in 1 cm quartz cells. Absorbance was set to 1 a.u. at $420 \mathrm{~nm}$, scan rate was $5 \mathrm{~s} / \mathrm{nm}$, and resolution $1 \mathrm{~nm}$. Four spectra between 200 and $600 \mathrm{~nm}$ were averaged and smoothed. Reference data (neat 5 peptide-coated silver particles) are published, ${ }^{35}$ and only differ in the number of repetitions (5 instead 4) and the spectral range (200 to $550 \mathrm{~nm}$ ). Estimation of the peptide secondary structure on the nanoparticle surface was done with $\mathrm{CDNN}^{71}$ software v2.1.

Magnetic measurements. Magnetic properties were 10 determined with an MPMS-XL Quantum Design SQUID magnetometer in the -5 to $+5 \mathrm{~T}$ and $1.8 \mathrm{~K}$ to $300 \mathrm{~K}$ ranges. Milligrams of powder samples were put in a gel cap for measurements. Magnetic susceptibility was recorded by applying a 500 to 10000 Oe static magnetic field. All data were corrected 15 for diamagnetic contributions of the samples and gel cap holder.

Cultivation with THP-1 macrophages. THP-1 cells were obtained from the Deutsche Sammlung für Mikroorganismen und Zellkulturen GmbH (DSMZ) stock collection. Cells were grown at $37{ }^{\circ} \mathrm{C}$ with $5 \% \mathrm{CO}_{2}$ in RPMI medium supplemented with $10 \%$ 20 fetal calf serum, $2 \mathrm{mM}$ L-glutamine, $10 \mathrm{mM}$ HEPES, $1 \mathrm{mM}$ pyruvate, $100 \mathrm{U} / \mathrm{mL}$ penicilline, and $0.1 \mathrm{mg} / \mathrm{mL}$ streptomycine. Differentiation into macrophage-like cells was done by adding $100 \mathrm{ng} / \mathrm{mL}$ phorbol-12-myristate-13 acetate. $^{72,73}$ Cell vitality after nanoparticle treatment was determined using the WST-1 25 assay (Roche Applied Biosystem) according to the manufacturers instruction with modifications to make the assay applicable for nanoparticle treated cells. Briefly, cells were seeded in a 96 well plate with a density of $1 * 10^{4}$ cells per well, differentiated, and incubated with nanoparticles (4 replicates/concentration). After ${ }_{30} 24$ or 48 hours, WST-1 reagent was added to the cells, the resulting solution centrifuged to remove the physically interfering nanoparticles and spectrophotometric evaluation was performed. The relative viability (\% viability in respect to untreated control cells) was calculated and expressed as a mean value and standard 35 error of the mean from at least 3 independent experiments. Homogeneous membrane integrity was determined by the LDH assay (Promega, Mannheim, Germany). Cells were grown as indicated for the WST test, and incubated with nanoparticles for 24 or 48 hours. Supernatants were analyzed with LDH assay 40 according to the manufacturer protocol. Results were expressed as the relative $\mathrm{LDH}$ release (\% of release in respect of Triton-X100 -lysed cells). The relative $\mathrm{LDH}$ release is a mean value and standard error of the mean of at least 3 independent experiments.

\section{Results}

\section{${ }_{45}$ Nanoparticle synthesis and structure}

The peptide-modified silver nanoparticles have been described previously. ${ }^{12}$ They can be dispersed in acidic aqueous solution as individual particles. At higher $\mathrm{pH}$, they aggregate due to lysine deprotonation. They can be silicified with tetraethoxyorthosilicate 50 (TEOS) yielding well-defined Ag/peptide@SiO 2 core-shell particles. ${ }^{35}$ The current report focuses on the doping of the silica layer with erbium(III) to prepare optically and magnetically active materials. Such particles could find application in readout systems with magnetic and optical detection, for example in anti55 counterfeiting, or in diagnostics.

To process the particles, the colloidal stability must be improved. Besides our standard silicification process, ${ }^{35}$ we have therefore also evaluated the modification of the outermost silica layer with aminopropyltriethoxysilane (APTES) for further 60 particle stabilization. Besides colloidal stabilization, APTES also enables additional functionalization of the particle surface.

Figure 1 shows representative TEM images of the erbiumdoped silver-peptide-silica nanoparticles (ESPN). ESPN grown in the presence of $0.3 \mathrm{~mol} \%$ of erbium (further on denoted as 0.3 ${ }_{65} \mathrm{~mol} \% \mathrm{Er}$, see experimental part for details) are spherical objects with a diameter between 25 and $28 \mathrm{~nm}$. After 48 hours the particle diameter increases by $c a .1-2 \mathrm{~nm}$ and the particles aggregate, similar to previously reported particles. ${ }^{35}$ Moreover, additional silica precipitation independent of the initial ESPN 70 formation occurs after 48 hours. However, upon redispersion and coating with APTES, the particles are well-dispersed, do not aggregate, and their size does not increase further.

Interestingly, samples grown with $0.3 \mathrm{~mol} \%$ of Er show a rather poor contrast between the silver core and the silica coating. ${ }_{75}$ This is somewhat surprising, because previous experiments ${ }^{35}$ have shown that the electron density contrast between the silver core and the silica shell (without Er) is quite strong. Although this is a qualitative argument, the current TEM data thus suggest that $\mathrm{Er}$ is incorporated into the silica shell, which sufficiently 80 increases the electron density to reduce the contrast between the silver core and the Er-doped silica shell.

The particles grown with $3 \mathrm{~mol} \%$ of Er (further on denoted as $3 \mathrm{~mol} \% \mathrm{Er}$ ) are different. After 24 hours the round particles with diameters between 25 and $30 \mathrm{~nm}$ have a 2-3 nm thin shell of a ${ }_{85}$ less electron-dense phase, presumably amorphous silica. Additionally, dark particles with a diameter of 4-5 nm can be observed in and on the silica shell. Bright field TEM therefore suggests that they are of different chemical composition than the silica, because they phase separate and have a higher contrast.

90 Like the particles grown with $0.3 \mathrm{~mol} \%$ of erbium, the silica layer on the nanoparticles grown with 3 mol\% increases to 4-5 $\mathrm{nm}$ after 48 hours and the smaller, presumably Er-rich, nanoparticles remain present. Moreover, TEM indicates that the number of these particles is relatively large and that they are 95 located both in and on the silica shell. It is however difficult to determine how many of the smaller particles are present on a silver nanoparticle. Again, upon coating with APTES, the size of the composite particles does not change.

For better characterization of the smaller nanoparticles in and 100 on the silica shell, high resolution TEM (HRTEM) was employed, Figure 2. Clearly, there is a (poly)crystalline core (silver, lattice spacing in the HRTEM images of $2.22 \AA$, (111) reflection) coated with an 1-2 nm amorphous layer (silica). HRTEM also shows that the smaller nanoparticles within the 105 silica shell are not amorphous, but crystalline, and have a diameter of up to $10 \mathrm{~nm}$. The lattice spacing of $3.21 \AA$ cannot be simply interpreted; it is however close to the A-phase erbium oxide (100) or B-phase erbium oxide (111) reflection. ${ }^{74}$ However, as no other lattice fringes could be observed, a final assignment is 110 not possible from HRTEM

High angle annular dark field scanning TEM (HAADF-STEM) confirms that the small particles observed in the silica shell are not silica. HAADF-STEM is sensitive to electron density (the atomic number $\mathrm{Z}$ ) and contrast scales approximately with $\mathrm{Z}^{2}$. 
Thus, the brighter a particle appears in HAADF-STEM, the higher the average $\mathrm{Z}$. HAADF-STEM clearly shows a high contrast indicative of a high average $\mathrm{Z}$ of the small particles. This confirms HRTEM, which suggests a crystalline structure (and 5 therefore something else than silica) for these small objects.
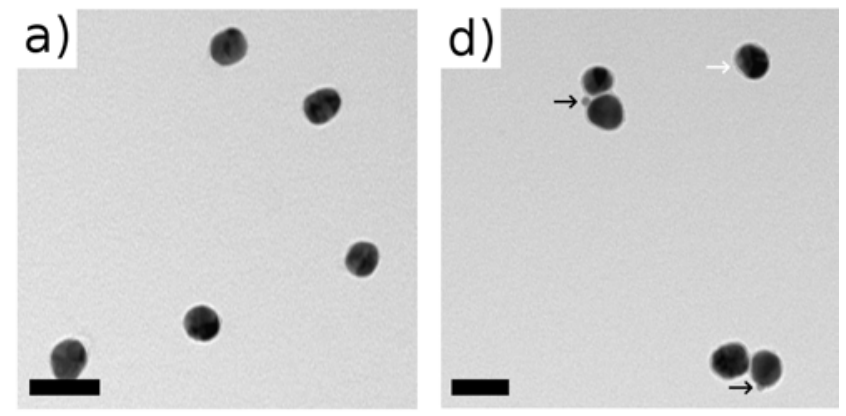

b)
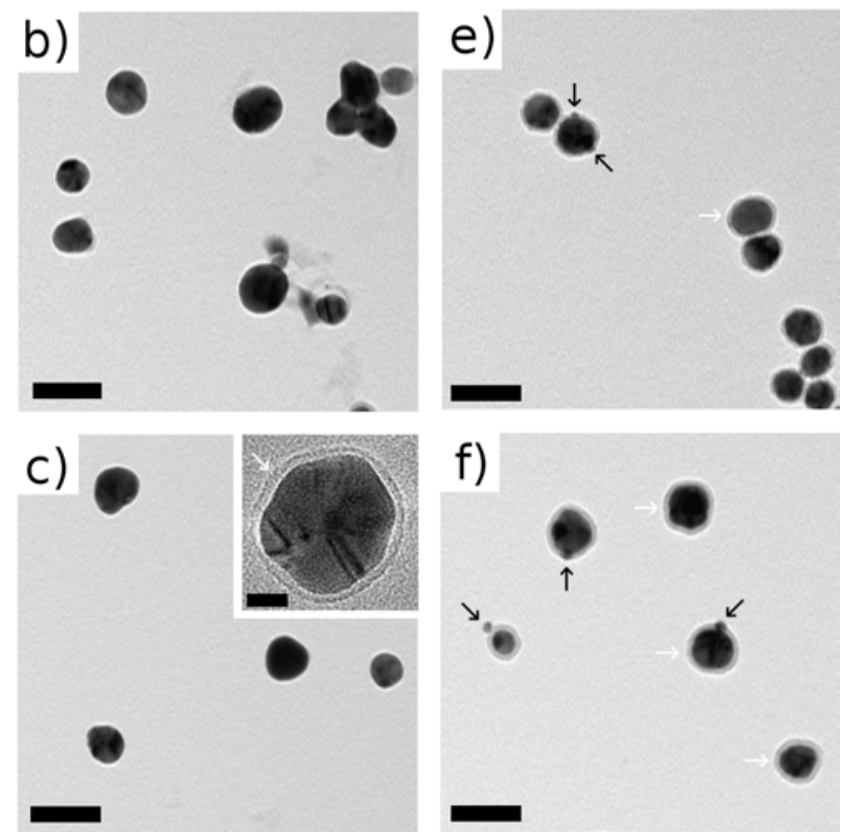

Fig. 1 TEM of different nanoparticles. Left column: ESPN grown with 0.3 mol\% Er, right column: ESPN grown with 3 mol\% Er. (a,d) after 24 hours, (b, e) after 48 hours and (c, f) after 72 hours and APTES coating.

$10 \quad$ Scale bar is $50 \mathrm{~nm}$. Inset: Zero loss energy filtered TEM image of a nanoparticle showing the silica shell which is normally barely visible.

Scale bar is $10 \mathrm{~nm}$. Black arrows points to Erbium-based nanoparticles; white arrows point to the growing silica layer. For TEM images of starting nanoparticles and nanoparticles without Er in the shell, see

15 Figure S1.

HAADF-STEM further shows that the number of small particles in the silica layer is higher than anticipated from bright field TEM (Figure 1). It does also confirm TEM in that also HAADF-STEM and HRTEM find smaller particles directly on 20 the silver particle surface and on the outside of the silica shell.

These findings are further supported by high-resolution scanning electron microscopy (HR-SEM, Figure S2), which finds small particles on the surface of the silver particles in the case of the samples grown with $3 \%$ of $\mathrm{Er}$ and no features in the sample 25 grown with $0.3 \%$. Moreover, energy dispersive X-ray spectroscopy (EDXS, Figure S4) clearly indicates the presence of Er in the sample with $3 \mathrm{~mol} \%$. The sample grown with only 0.3 mol\% does not exhibit a significant Er signal, presumably due to poor signal to noise ratio or too low excitation efficiency of the 30 Er transitions (EDX, Figure S5).

In summary, at low erbium concentration, TEM, HRTEM, and STEM suggest that erbium may be simply included in the silica layer as a dopant. At higher concentration, crystalline nanoparticles form, either directly on the silver nanoparticle 35 support (that is, in the silica layer) or on the silica layer.
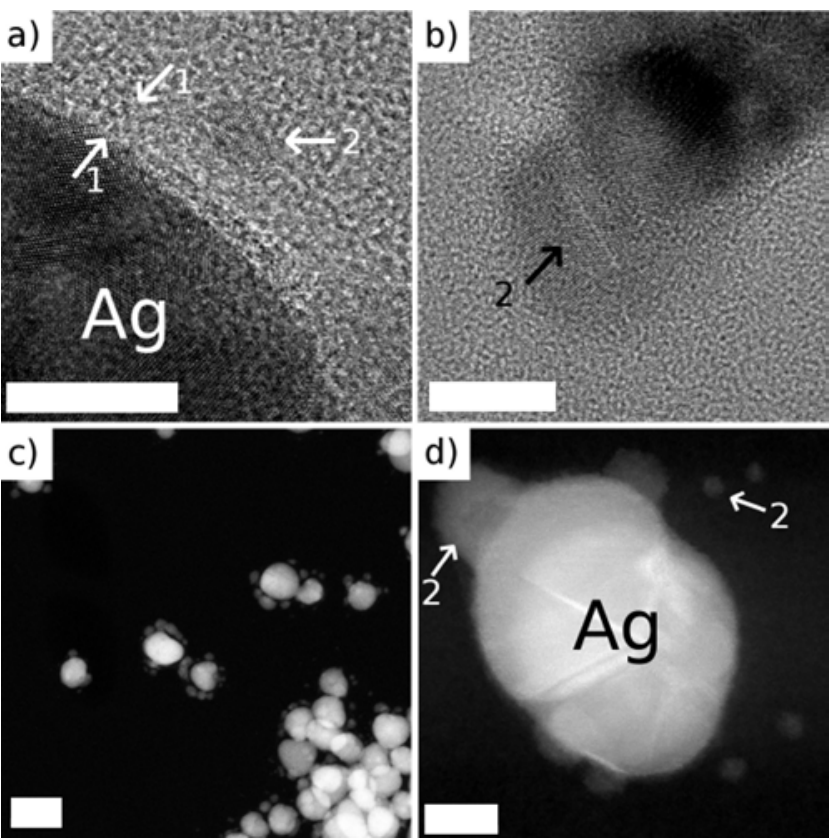

Fig. 2. HRTEM and HAADF-STEM images of ESPN (3 mol\%). (a) HRTEM image of a silver/silica boundary. Arrows (1) indicate the silica layer; arrow (2) denotes an erbium containing nanoparticle. (b) HRTEM 40 image of an erbium-containing nanoparticle (arrow). (c) Low and (d) high magnification HAADF-STEM images. Arrows indicate erbium-

containing nanoparticles on and in the silica layer, respectively. Scale bars are 10 (a, b, d) and $50 \mathrm{~nm}$ (c)

Figure 3 shows synchrotron X-ray diffraction (sXRD) data of ${ }_{45}$ the ESPNs and a reference sample. ${ }^{12}$ The crystalline silver nanoparticle core is preserved in all particles, as indicated by the broad silver (111) and (200) reflections (JPCPS 04-0783). In the erbium-doped particles, additional reflections are visible. Figure 4 shows a tentative indexing for the supplementary phases, which 50 can be attributed to B-phase ${ }^{74}$ (ICSD 160230) and C-phase ${ }^{75}$ (ICSD 27774) erbium oxide. ${ }^{76}$ Due to the low signal intensity, phase quantification and unambiguous assignment is at this stage not possible. In spite of this SXRD supports TEM, SEM, and EDXS in that there is clear evidence for an additional component ${ }_{55}$ in the hybrid particles. SXRD, however, also shows that the same Er species that, presumably, forms the small particles observed in the sample grown with $3 \%$ of $\mathrm{Er}$ is also present in the samples grown with $0.3 \%$. This is thus the first evidence that the same products (although possibly in quite different concentrations) are 60 obtained in both cases. 


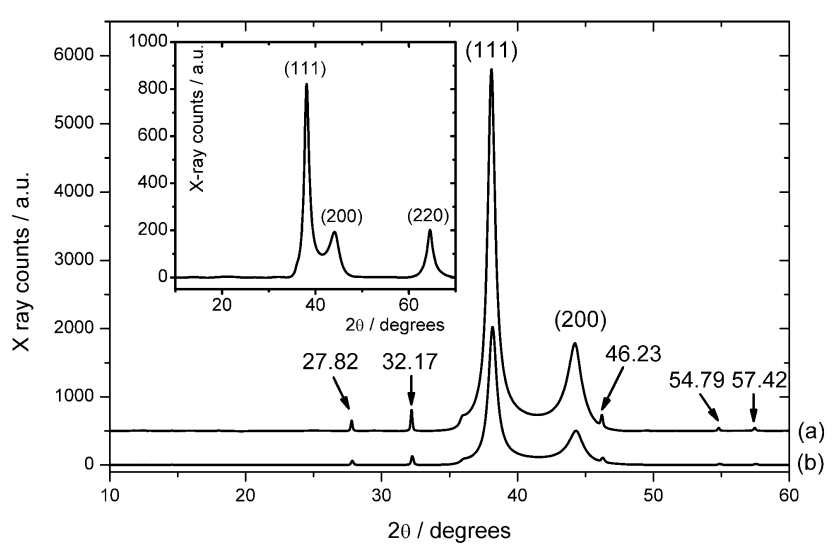

Fig. 3 sXRD patterns of (a) ESPN prepared with 0.3 mol\% of Er and (b) ESPN prepared with 3 mol\% of Er. Arrows point to new reflections; numbers scattering angles in $2 \theta$. Inset: sXRD pattern of peptide-coated silver nanoparticles grown without Er and without silica layer. ${ }^{12}$

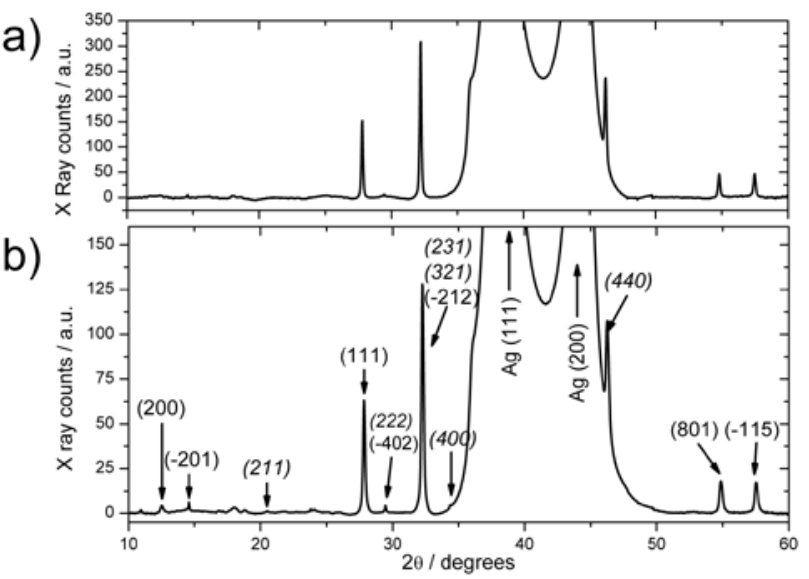

Fig. 4: sXRD diffractogram of ESPN prepared in the presence of 0.3 mol\% $\mathrm{Er}$ (a) and $3 \mathrm{~mol} \% \mathrm{Er}$ (b) with indexing for $\mathrm{Er}_{2} \mathrm{O}_{3}$ B-phase (ICSD 160230) and $\mathrm{Er}_{2} \mathrm{O}_{3}$ C-phase (ICSD 27774, in italics). For more details, see Figure S3, Table S1, and experimental section.

As microscopy is a very local technique, and thus a comprehensive and correct sample description based on this single technique is difficult, we performed complementary smallangle X-ray scattering (SAXS) measurements. Figure 5 shows ${ }_{15}$ SAXS data of both erbium-doped samples. They show very similar scattering curves, with a clear first minimum at $c a . q=0.3$ $\mathrm{nm}^{-1}$, and a weaker one at around $q=0.6 \mathrm{~nm}^{-1}$. The curve shows a reasonable Porod behavior, that is, I $(q)$ scales with $q^{-4}$ for $q>1$ $\mathrm{nm}^{-1}$. This indicates a sharp interface of the nanoparticles with 20 their surroundings. The minima indicate a moderate polydispersity, which is rather surprising for a core-shell system. Data were fitted from 0.1 to $2 \mathrm{~nm}^{-1}$, as the curves do not exhibit distortions due to attractive forces between the particles or the presence of aggregates. In both cases, curve fitting using a ${ }_{25}$ polydisperse core and a monodisperse shell leads to virtually identical results, that is, an average silver core radius of $12.5 \pm$ $0.2 \mathrm{~nm}$, a polydispersity of $20 \%$, and a shell of $1.2 \pm 0.2 \mathrm{~nm}$. This result is slightly larger than the initial silver particles ${ }^{12}$ which have a radius of $9.3 \pm 3.6 \mathrm{~nm}$, see Figure S1. On the other hand, 30 a radius of $12.5 \mathrm{~nm}$ corresponds fairly well with TEM and SEM, see above. There are two possible causes for this deviation. (1) The fitting process only partly describes the particle structure and another approach may be better ${ }^{77}$ or (2) the Er affects not only the properties of the final nanoparticles but also changes the 35 formation process. At the moment, however, we assign this difference to batch-to-batch variations in the synthesis.

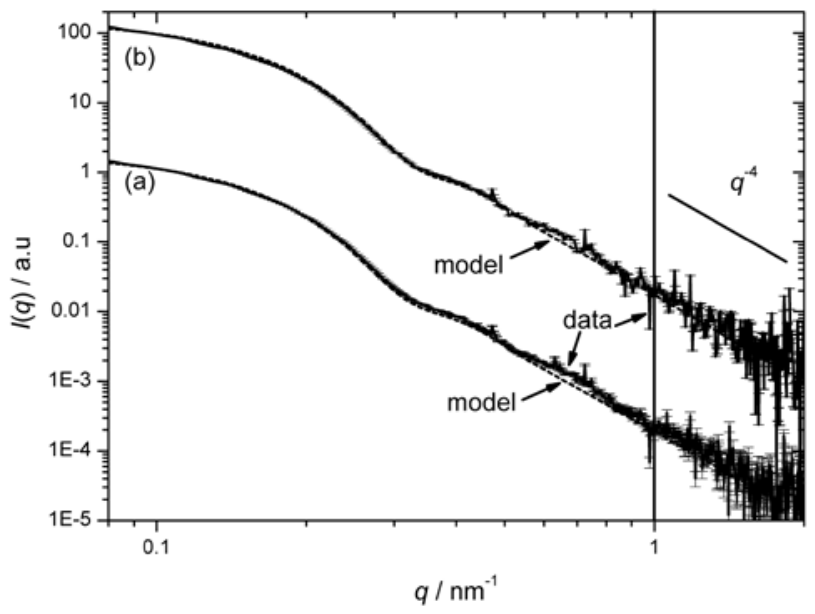

Fig. 5: Fitted SAXS curves of ESPN prepared in the presence of (a) 0.3 $\mathrm{mol} \% \mathrm{Er}$ and (b) $3 \mathrm{~mol} \% \mathrm{Er}$. Data are shifted vertically for clarity. The 40 Porod region is indicated with a vertical straight line. Experimental data and fit at lower $q$ values overlap and can not be distinguished.

Because regular core-shell models (that is: two distinct layers, with homogeneous electron densities) may not be able to fully account for the rather complex structure of the ESPN, we have ${ }_{45}$ performed a total non-negative least square (TLNNS) analysis of the experimental data. In brief (see experimental part for details), a nanoparticle population with different characteristics like size, size distribution, etc. is generated in the computer. The corresponding scattering curve is calculated and matched with 50 experimental data. Then, the nanoparticle population is modified dynamically to fit the experimental data. For complex systems this has the advantage to limit the amount of fitting parameters and to not generate non-processable models. Figure 6 shows the different fits with the corresponding residuals, and the volume55 averaged size distributions generated from the experimental scattering curves of our samples.

In both cases the overall particle diameter is around $27 \mathrm{~nm}$ with a polydispersity of ca. $25 \%$. This is in good agreement with the previous SAXS data of the undoped nanoparticles, where an 60 overall size of around $28 \mathrm{~nm}$ was determined, Figure S5. ${ }^{35}$ Interestingly, the TLNNS analysis clearly indicates two $(0.3 \% \mathrm{Er})$ or three $(3 \% \mathrm{Er})$ different particle populations. The size distribution appears to depend on the initial erbium alkoxide concentration. As the initial silver nanoparticles are essentially 65 monodisperse and monomodal, we assign the smaller sizes to the erbium-based nanoparticles found in HRTEM and STEM.

Figure 7 shows the evolution of the experimental pair distribution function $\mathrm{P}(\mathrm{r})$ and the deconvolution in a radial electron density function $\rho(\mathrm{r})$ calculated from the SAXS data. The 70 advantage of this approach is that no specific knowledge of the sample structure is required. Simulated and experimental $\mathrm{P}(\mathrm{r})$ are in a good agreement for both samples. $\mathrm{P}(\mathrm{r})$ is typical of a coreshell structure, thereby again supporting electron microscopy.

The radial electron densities $\rho(\mathrm{r})$ of both systems ( 0.3 and 3 $75 \mathrm{~mol} \% \mathrm{Er}$ ) are virtually identical and exhibit three regions: first, a 
plateau until $2 \mathrm{~nm}$, then an increase until ca. $8 \mathrm{~nm}$, and finally a decrease to zero at around $14 \mathrm{~nm}$. The overall $\rho(\mathrm{r})$ curve is rather atypical for core-shell systems, but can be explained by the special particle structure: the core electron density is due to 5 silver. The increasing electron density at the silver particle surface clearly indicates the presence of an erbium species close to the silver surface. Indeed, the $\rho(\mathrm{r})$ curve does not indicate an Er layer or Er particles, but the increase in electron density at ca. 6-8 $\mathrm{nm}$ may be caused by the presence of a significant amount of ${ }_{10} \mathrm{Er}$ (doped into the silica) relatively close to the silver particle surface. As the silicification reaction proceeds, the reaction mixture is depleted of Er and the average $\mathrm{Z}$ decreases towards the outer surface of the particles.
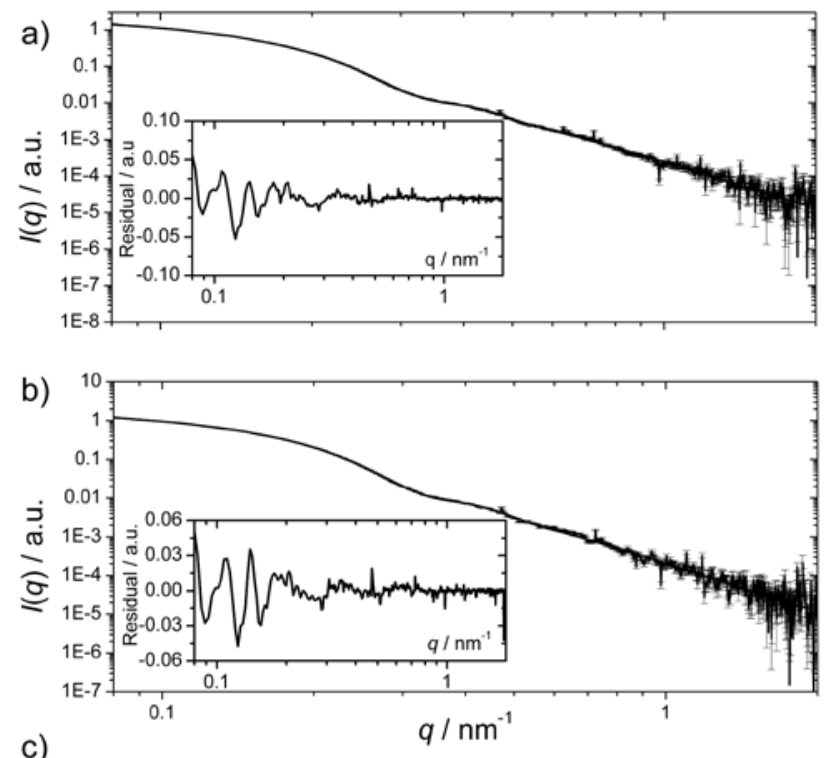

c)

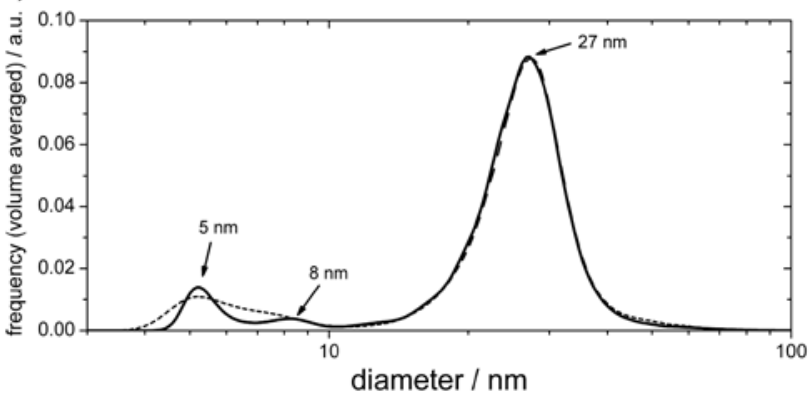

15 Fig. 6: Fitted SAXS curves of ESPN prepared in the presence of (a) 0.3 mol\% Er and (b) 3 mol\% Er using a TNNLS model (both insets: residuals in absolute difference). c) Particle size distributions obtained from the TNNLS analysis (dotted: $0.3 \mathrm{~mol} \% \mathrm{Er}$, straight: $3 \mathrm{~mol} \% \mathrm{Er}$ ). For data on the earlier core/shell particles, ${ }^{35}$ see Figure S6. For a comparison with other SAXS models, see Figure S7.

As a result, SAXS confirms TEM in the sense that the electron density of the samples grown with $0.3 \mathrm{~mol} \%$ of Er is relatively high in the silica films even without any particulate Er-rich component. This may account for the poor contrast and the 25 apparent absence of the erbium/silica layer in the TEM bright field images of these samples, Figure 1. Finally the decrease towards 0 at ca. $14 \mathrm{~nm}$ is due to the fact that the outer particle shell is reached. The $14 \mathrm{~nm}$ correspond fairly well to the particle radius obtained from SAXS and electron microscopy.

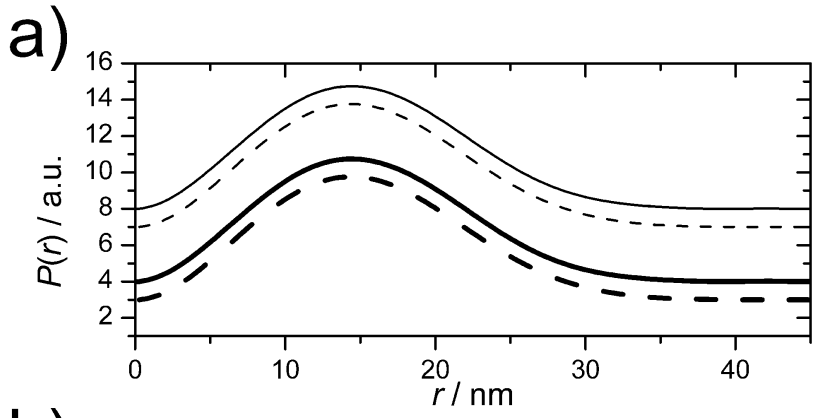

b)

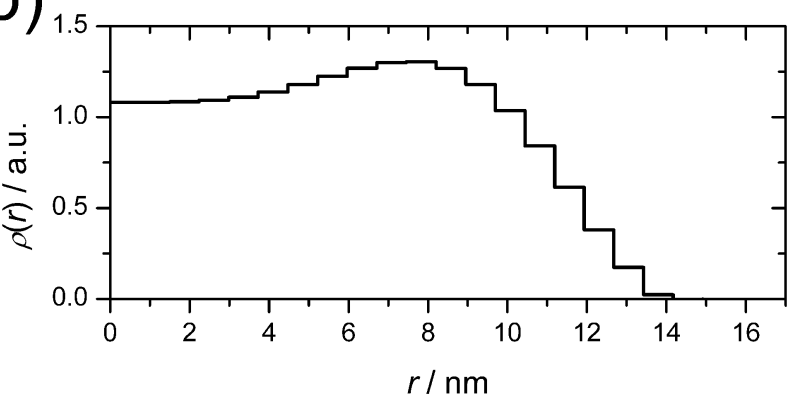

Fig 7: Experimental $P(r)$ determined from the scattering curve via GIFT (solid line) and by DECON fitting (dashed line) for ESPN prepared in the presence of $0.3 \mathrm{~mol} \%$ (thin line) and $3 \mathrm{~mol} \%$ (thick line) of erbium.

Curves were vertically shifted for clarity. (b) Electronic pair distribution

35 function $\rho(r)$ calculated from the experimental $P(r)$ function. Both $\rho(\mathrm{r})$ are identical irrespective to the starting concentration of erbium precursor.

\section{Optical properties}

Metal nanoparticle shape, size, and dielectric environment can be characterized using UV-Vis spectroscopy. Figure 8 shows the ${ }_{40} \mathrm{UV}-\mathrm{Vis}$ spectra of the nanoparticles after 48 hours of reaction and subsequent coating with APTES, for 0, 0.3, and $3 \mathrm{~mol} \%$ of Er. For comparison, the spectrum of the neat peptide-coated silver nanoparticles $^{12}$ is also shown. Peptide-coated nanoparticles exhibit maxima at 352, 376, 414, 442, and $497 \mathrm{~nm}$, which are due 45 to scattering and plasmon resonance of single nanoparticles. ${ }^{78,79}$ The band at $497 \mathrm{~nm}$ indicates that some particles are distorted. ${ }^{78}$ The bands at $600 \mathrm{~nm}$ are barely visible, ${ }^{79}$ indicating that the particles are well dispersed in the medium. ${ }^{12}$

Upon silicification and erbium addition, the absorption 50 maximum shifts from $415 \mathrm{~nm}$ (only with silica shell, no erbium) to 426 (0.3 mol\% Er) and $437 \mathrm{~nm}$ (3 mol\% Er). These findings are a direct consequence of the Mie theory, but also a strong indication that erbium is included in the structure, even though EDX was not able to detect it unambiguously in the case of 0.3 $55 \mathrm{~mol} \%$ of Er (Figures S4 and S5). We currently speculate that erbium included in the silica layer changes the medium dielectric constant, ${ }^{80}$ resulting in a pronounced red-shift upon Er doping.

At $3 \mathrm{~mol} \%$ of erbium, an additional band at $547 \mathrm{~nm}$ appears. This band cannot be attributed to silver nanoparticle, silica, or ${ }_{60}$ "doped silica" but matches fairly closely with some $\mathrm{Er}_{2} \mathrm{O}_{3}$ absorption bands. ${ }^{81}$ This supports SEM, TEM, EDX, XRD, and SAXS. Altogether, the data show that we have created a hybrid material based on silver, a small peptide, and silica, similar to our previous report, ${ }^{35}$ but with the added interesting component of an ${ }_{65}$ Er-rich silica shell or Er-rich nanoparticles, possibly $\mathrm{Er}_{2} \mathrm{O}_{3}$ or a related phase, depending on the initial Er concentration. It must be noted at this point that the deconvolution into individual 
bands, similar to the one done for the earlier, undoped particles, ${ }^{12}$, 35 fails if Er is present. We can therefore at the moment not comment on further details of the UV/Vis spectra.

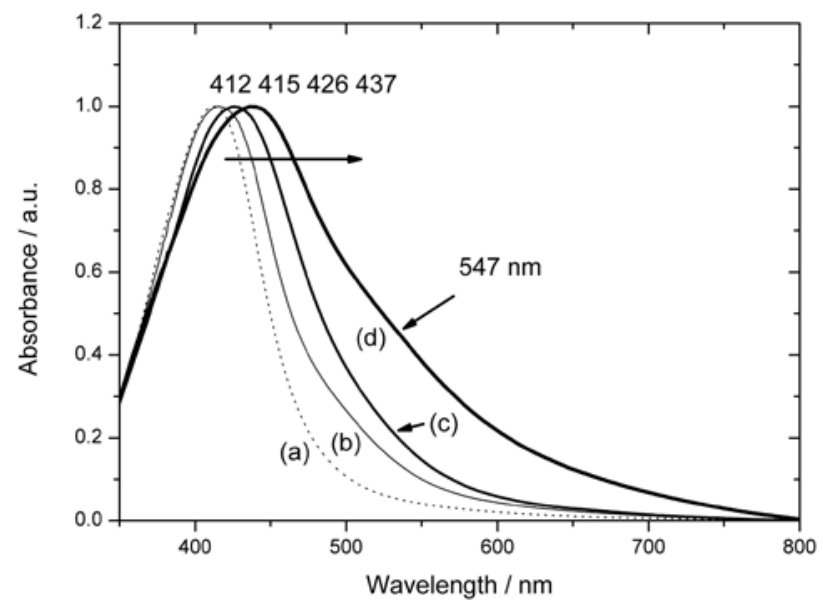

5 Fig. 8: UV/Vis spectra of nanoparticles. (a) Pure silver nanoparticles (no silica shell) ${ }^{12}$, (b) nanoparticles coated with only silica (no Er), (c) 0.3 mol\% of Er, (d) 3 mol\% of Er. For a discussion of the spectral properties versus reaction time, see Figure S7.

Figure 9 shows representative Raman spectra of the 10 nanoparticles. The peptide-coated nanoparticles (no silica or erbium) show a complex spectrum composed of a variety of bands arising from carbonyl, amide, and aliphatic vibrations. The same bands can be observed after silicification, indicating that the peptide is intact (in terms of conformation) even after inclusion 15 into the silica layer. ${ }^{35}$ In contrast, Raman spectra of the samples grown with erbium show that the overall spectrum is conserved, but the peptide conformation is modified. A new band at 1733 $\mathrm{cm}^{-1}$ and the disappearance of the band at $925 \mathrm{~cm}^{-1}$ indicate that the peptide secondary structure has changed, possibly due to 20 complexation with erbium ions or an interaction with the newly formed inorganic phase observed in XRD and HRTEM.

As the Er-O band is, for example, in close vicinity to the silica $\gamma(\mathrm{Si}-\mathrm{O})$ band at $460 \mathrm{~cm}^{-1},{ }^{22}$ the erbium oxide phase cannot be assigned unambiguously. Diagnostic bands at lower 25 wavenumbers $\left(<200 \mathrm{~cm}^{-1}\right)$ are not accessible with our instrumentation. Altogether, Raman spectroscopy nevertheless confirms the presence of the peptide on the nanoparticle surface and suggests that the peptide interacts in some way with the erbium species during and after silica shell formation. Moreover,

30 Raman spectroscopy also provides clear evidence of the presence of a silica layer, thus supporting SAXS and TEM experiments.

Figure 10 shows the CD spectra of the hybrid particles. Above $350 \mathrm{~nm}$, all spectra are essentially identical and present a number 35 of Cotton effects, which are difficult to assign clearly. They are, however, at least shifted by $20 \mathrm{~nm}$ compared to the Cotton effects observed in the pure peptide-coated silver nanoparticles. ${ }^{35}$ The shift is due to the change in the dielectric environment of the nanoparticles, which has been mentioned previously.

40 Below $320 \mathrm{~nm}$ the CD signal predominantly arises from the organics at the nanoparticle surface. Comparison of the CD signals of the pure nanoparticle (especially the organic signal, see Table S2) and the hybrid particle without erbium only shows marginal differences, which again confirms that the silicification
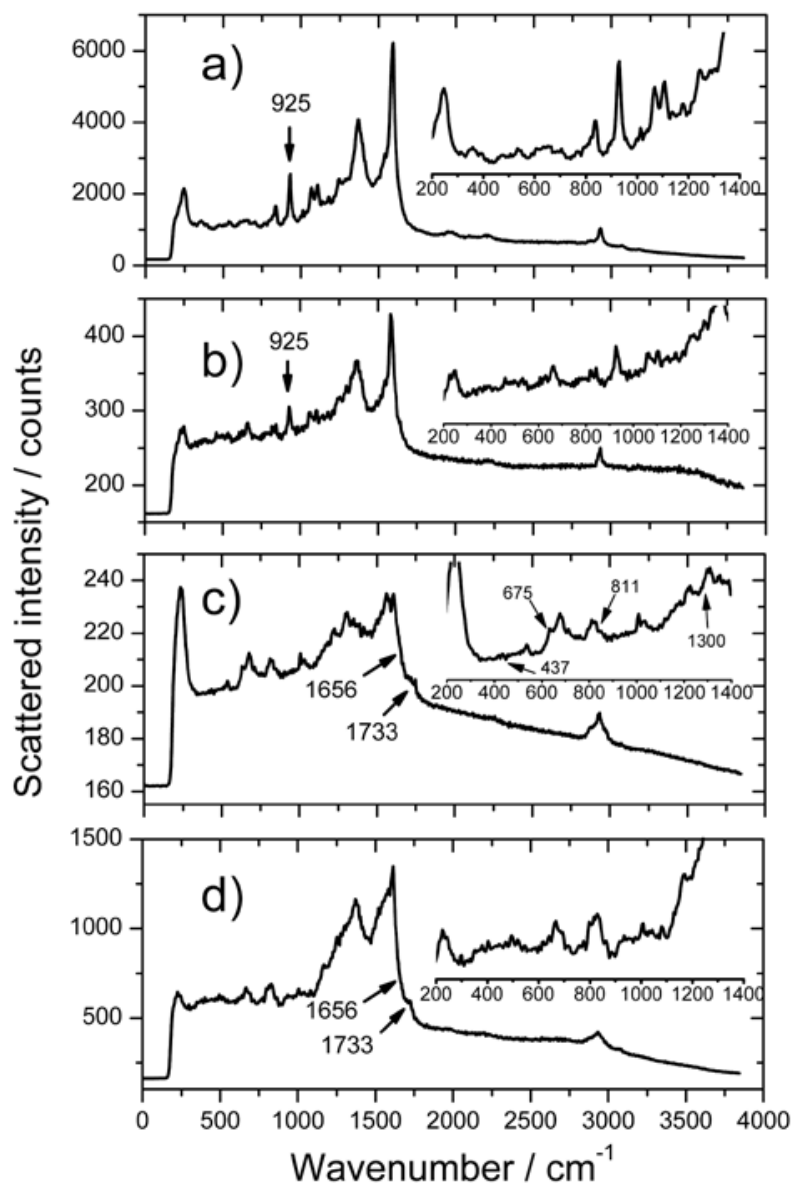

45

Fig.9 Raman spectra of nanoparticles. (a) Pure peptide-coated nanoparticle (no silica), (b) 0 mol\% Er (pure silica shell), (c) $0.3 \mathrm{~mol} \%$ Er, and (d) 3 mol\% Er. Insets are expanded views of the low frequency area with the spectral signals of the inorganics. The hump at $1656 \mathrm{~cm}-1$ is 50 caused by the carboxylate at the peptide $\mathrm{C}$ terminus. ${ }^{35}$ For a detailed view on the low frequency region, see supporting information, Figure S10.

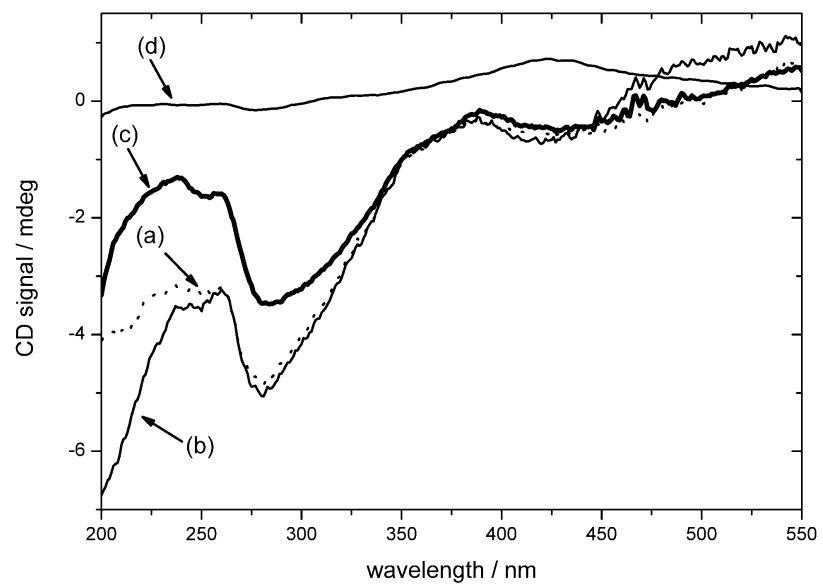

Fig. 10 CD spectra of nanoparticles. (a) $0 \%$ Er (silica shell only), (b) $0.3 \% \mathrm{Er}$, (c) 3\% Er and (d) neat nanoparticles (without silica shell). All 55 spectra were recorded in the same conditions; absorbance was set to 1 at $420 \mathrm{~nm}$ and the $\mathrm{CD}$ spectra thus have a different intensity in the range shown here

process does not alter the secondary structure of the peptide. ${ }^{35}$ Slightly more pronounced changes can be found as soon as 60 erbium is introduced in the system. This can be correlated with a change in the secondary structure of the peptide, as inferred from 
Raman spectroscopy.

\section{Magnetic properties}

Figure 11 shows the temperature dependence of both magnetic 5 susceptibility and $\chi T$ curve of the ESPN between $1.8 \mathrm{~K}$ and 300 $\mathrm{K}$. The susceptibility variation of the particles differs by one order of magnitude, which is in good agreement with the $\mathrm{Er}$ concentration. Both Er-doped samples show an identical paramagnetic behavior with a hump at around $50 \mathrm{~K}$. Accordingly, 10 the peak observed below $50 \mathrm{~K}$ in the $\chi T$ curves indicates the presence of a magnetic component. Above $80 \mathrm{~K}$, the $\chi T$ products are linear and almost constant at $0.049 .10^{-4}$ and $0.50 .10^{-4} \mathrm{~K}$ emu $\mathrm{g}^{-1}$ for the $0.3 \mathrm{~mol} \% \mathrm{Er}$ and $3 \mathrm{~mol} \%$ Er samples, respectively. Such quasi-constant values suggest a magnetic ground state well 15 separated from the excited J multiplets.

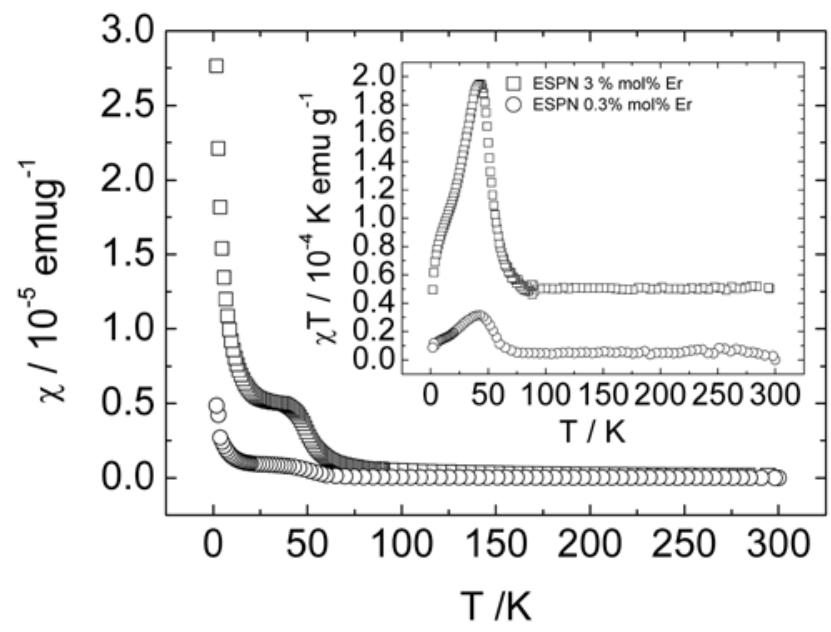

Fig. 11. Temperature dependence of the magnetic susceptibility of the

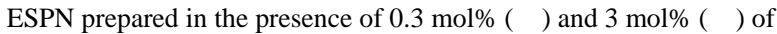
erbium.

20 To evaluate the magnetic state of both hybrid structures, the magnetization variation was measured vs. the applied field at 1.8 K, Figure 12. Again, both samples exhibit paramagnetic behavior, with no hysteresis and small saturation values $\mathrm{Ms}_{\mathrm{s}}=$ $0.08 .10^{-4}$ and $0.3 .10^{-4} \mu_{\mathrm{B}} / \mathrm{g}$ for 0.3 and $3 \mathrm{~mol} \%$ Er samples, 25 respectively.

Magnetic measurements thus suggest that the Er content is very low, as shown by the small Curie constants deduced from the $\chi \mathrm{T}$ product at room temperature $(\mathrm{C} \approx \chi \mathrm{T}$ at $300 \mathrm{~K})$. For $\mathrm{Er}^{3+}$ ions in a ${ }^{4} \mathrm{I}_{15 / 2}, \mathrm{~g}_{\mathrm{J}}=6 / 5$ ground state, a value of $\mathrm{C}=11.5 \mathrm{~K}$ emu $30 \mathrm{~mol}^{-1}$ would be expected. ${ }^{83}$ Thus, the experimental values would correspond to ca 0.007 and 0.0007 wt $\%$ of Er in the products (0.02 to $\left.2.10^{-3} \mathrm{~mol} \%\right)$ The same order of magnitude can be deduced from the saturation magnetization values (expected $\mathrm{Ms}_{\mathrm{S}}$ $=15 / 167.28=0.09 \mu \mathrm{B} / \mathrm{g})^{84}$ with quite large uncertainties due to 35 the strong diamagnetism of the samples.

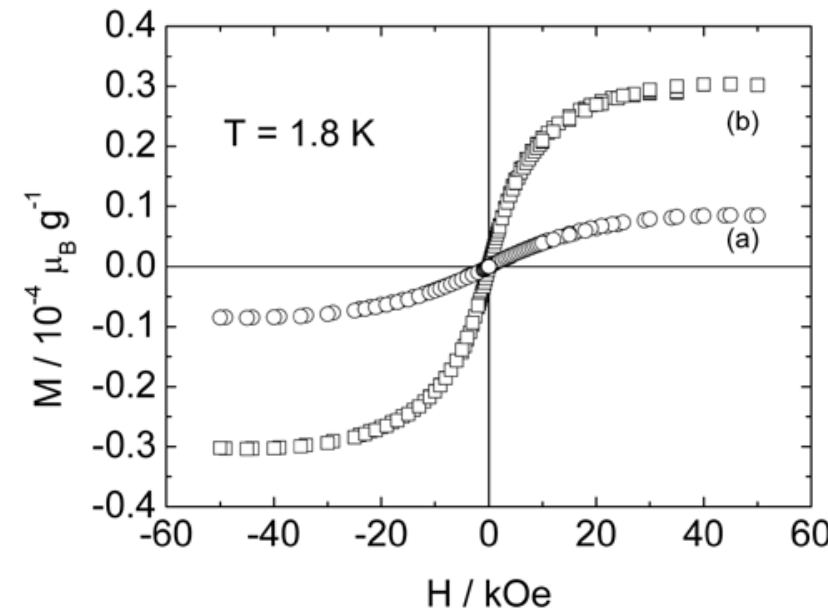

Fig. 12: Magnetization variation versus applied field, recorded at $1.8 \mathrm{~K}$ for nanoparticles prepared in the presence of (a) $0.3 \mathrm{~mol} \%$ and (b) 3 $\mathrm{mol} \%$ of erbium. Percentages correspond to the weight fraction of erbium 40 in the starting mixture. The data were corrected for strong diamagnetic contributions evaluated from the negative slope of the experimental curves that are linear at high fields.

Based on the synthesis process, we can rule out that the 45 magnetic signal arises from systems like metallic erbium, complex alloys based on erbium-silicon or erbium-silicon-silver. The presence of erbium silicates is possible. Unfortunately, only a limited amount of magnetic data on rare earth (specifically erbium) silicates exist. ${ }^{85}$ To our best knowledge, only $\mathrm{Er}_{2} \mathrm{Si}_{2} \mathrm{O}_{7}$

50 was characterized; it behaves antiferromagnetically with an ordering temperature $\mathrm{T}_{\mathrm{N}}=1.9 \mathrm{~K}$ and $2.5 \mathrm{~K}$ for the $\mathrm{D}$ and $\mathrm{C}$ structural types, respectively. ${ }^{86}, 87 \mathrm{Er}_{2} \mathrm{O}_{3}$, which has been discussed above, exhibits a non-collinear antiferromagnetic structure. ${ }^{88,89}$ Its ordering Néel temperature depends on particle 55 size but was not found exceeding the bulk value of $3.4 \mathrm{~K} .{ }^{90}$

Nevertheless, the hump of the susceptibility observed around $50 \mathrm{~K}$ was already observed for $\mathrm{Gd}_{2} \mathrm{O}_{3}$ nanoparticles embedded in $\mathrm{SiO}_{2}$ and was ascribed to the presence of superparamagnetic nanoparticles of the sesquioxide, the width of the hump 60 suggesting a distribution of blocking temperatures. ${ }^{91}$ The fact that the present ESPN could involve a mixture of antiferromagnetic and superparamagnetic $\mathrm{Er}_{2} \mathrm{O}_{3}$ nanoparticles is consistent with the experimental magnetic features. Magnetic measurements thus agree with X-ray and electron microscopy in 65 that they present clear evidence for an Er-containing species, although a structure assignment cannot be given.

\section{Biological and toxicological properties}

Silver nanoparticles and silver core-shell structures are important tools for analytical chemistry, for example as sensors 70 or reporting systems in living cells. ${ }^{20,92}$ If our particles are to be used in diagnostics, the biological and toxicological parameters must be known.

Figure 13 shows the viability of THP-1-derived macrophages incubated with different nanoparticles (WST-1 assay). After 24

75 hours of incubation, the silica-coated nanoparticles (with or without Er) do not significantly impair the viability of the macrophages. Interestingly, at the highest concentration (50 $\mu \mathrm{g} . \mathrm{mL}^{-1}$ ), the presence of erbium in the particles significantly 
improves the tolerance of the cells towards the nanoparticles compared to the silica-coated, but Er-free nanoparticles.

After 48 hours of exposition, the findings are essentially the same. Again the Er-doped silica shells seem to improve the 5 tolerance of the THP-1 cells compared to the Er-free nanoparticles. In spite of this, the tolerance is lower after 48 than after 24 hours. Moreover, APTES coatings do not significantly change the toxicity of the particles; both with and without APTES coating, the Er-free, but silica-coated nanoparticles elicit a 10 stronger response than the Er-doped particles. Finally, particles without a silica coating are the most toxic and already at low concentrations reduce the cell viability. In summary, the WST-1 assay thus suggests that the Er-doping (i) stabilizes the silica shell or (ii) has another beneficial effect on the THP-1 macrophages. In 15 contrast, APTES-modification of the surface appears of minor importance, while the presence or absence of a silica shell is important for the biological response.
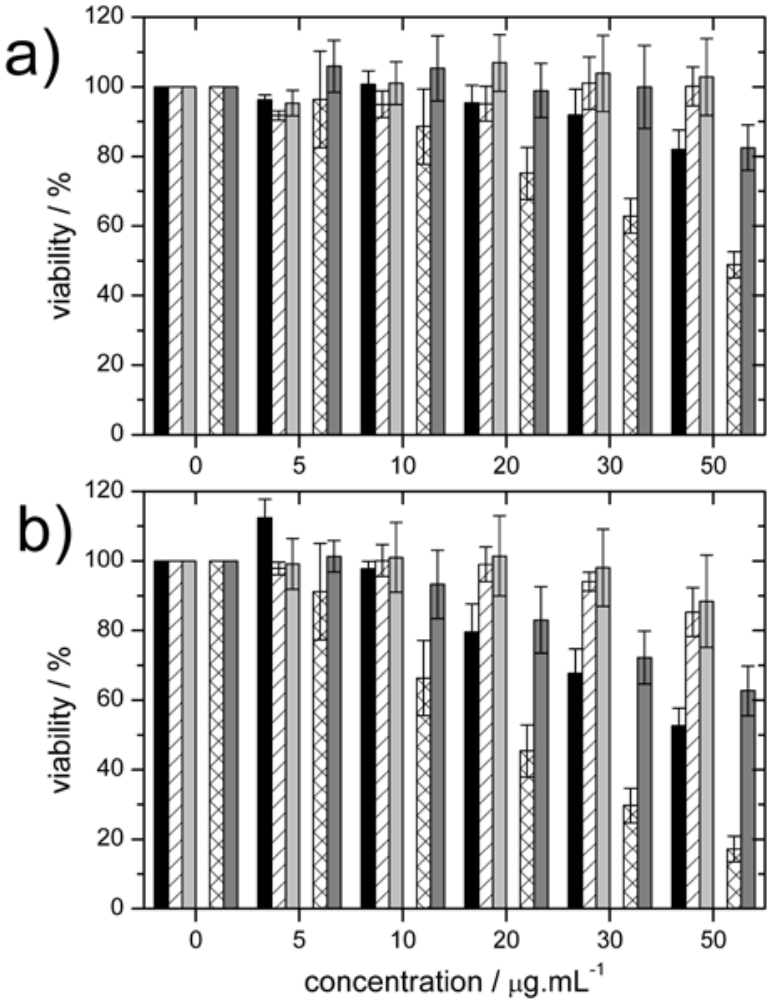

\begin{tabular}{|ll|}
\hline with APTES coating & without APTES coating \\
ESPN + 0\% Er & $\times$ AgCKK \\
$\square Z$ ESPN + $0.3 \mathrm{~mol} \% \mathrm{Er}$ & $\square$ Ag/peptide@SiO \\
$\square$ ESPN + 3 mol\% Er & \\
\hline
\end{tabular}

Fig. 13 Viability assay of macrophages differentiated from THP-1 cells 20 exposed for a) 24 hours or b) 48 hours to peptide-coated nanoparticles, silica-coated nanoparticles, and Er-doped nanoparticles. Silica-coated nanoparticles were further modified with APTES to improve colloidal stability. Ag/peptide@SiO ${ }_{2}$ are nanoparticles as described in our previous paper, that is, without an APTES layer..$^{35}$ AgCKK are nanoparticles 25 without silica coating. ${ }^{12,93,94}$

Figure 14 shows the results of an LDH assay after 24 and 48 hours. LDH levels in the cell culture medium are indicative of the membrane integrity, and thus macrophage integrity. The LDH assay confirms the WST-1 assay and detects a dose- and time-
30 dependent cytotoxicity, which is clearly influenced by the presence of a protective silica layer. Particles without silica shell are significantly more cytotoxic. Depending on the incubation time these particles show a significant effect already at $10 \mu \mathrm{g} . \mathrm{mL}$

1 . In contrast, silica-coated nanoparticles hardly show an effect up 35 to $50 \mu \mathrm{g} . \mathrm{mL}^{-1}$ at 24 hours of incubation.

Consistent with Figure 13, the LDH data show a lower LDH leakage after 48 hours if Er-doped particles and not just silicacoated or silica-APTES coated nanoparticles are used. This again suggests a stabilizing effect of Er on the silica shell. Further 40 experiments are however necessary to confirm this.
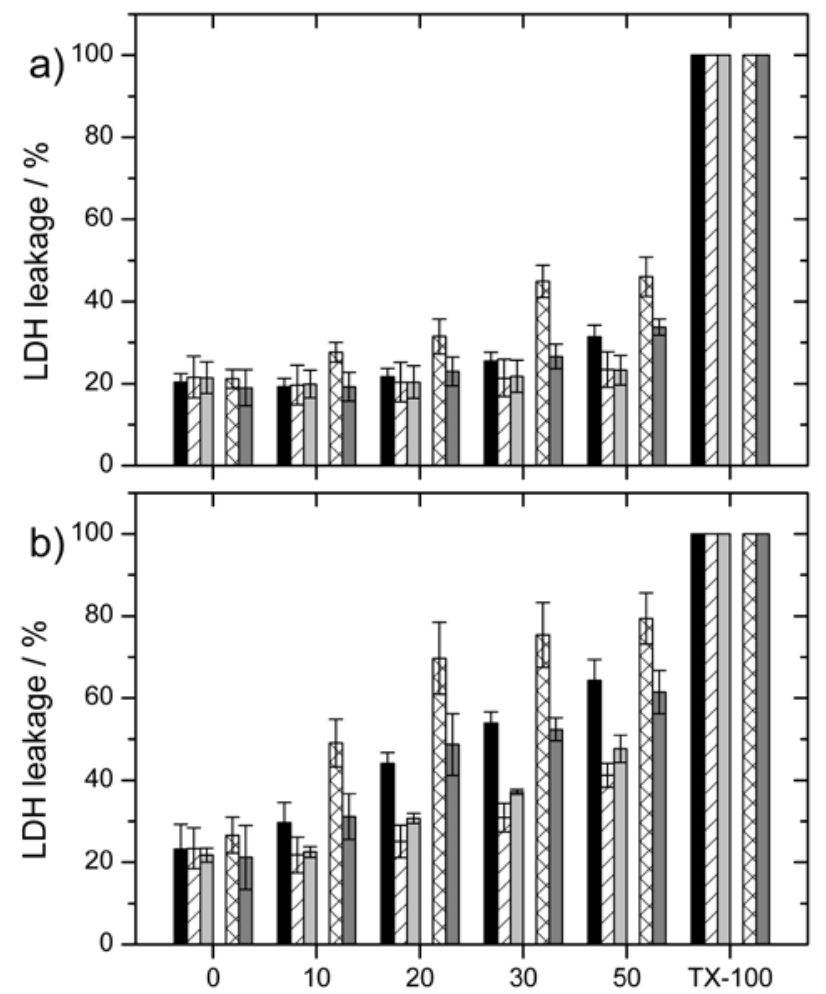

Nanoparticle concentration / $\mu \mathrm{g} \cdot \mathrm{mL}^{-1}$

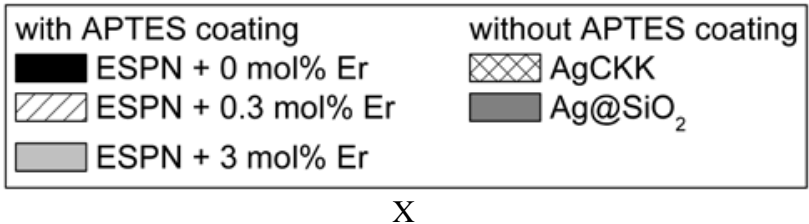

Fig. 14 LDH leakage experiment of macrophages differentiated from 45 THP-1 exposed to the ESPN for (a) 24 hours or (b) 48 hours. The gap between the columns separates the ESPN results from the model nanoparticles.. ${ }^{12,35,93,94}$ TX-100 is Triton X-100.Ag/peptide@SiO ${ }_{2}$ are nanoparticles as described in our previous paper, that is without a APTES layer; ${ }^{35}$ AgCKK are nanoparticles without silica coating. ${ }^{12}$

50

\section{Discussion}

We have synthesized new nanoparticles with a complex structure. At low Er concentrations in the initial reaction mixture, the peptide-coated silver nanoparticles ${ }^{35}$ are coated with a thin ${ }_{55}$ silica layer, which appears to be Er-rich. This can be inferred 
from TEM, STEM, Raman, XRD, SAXS, and magnetic measurements (Figures 1-7, 9, 11-12). In contrast, TEM, STEM, and SAXS (Figures 1, 2, 5-7) of the samples synthesized with higher Er concentrations in the precursor solution show that these 5 nanoparticles contain ca. $5 \mathrm{~nm}$ particles both in and on the silica shell. HRTEM, STEM, and XRD (Figures 2, 3 and 4) show that these small particles are crystalline, but the structure can at the moment not be assigned unambiguously. Nevertheless, Raman spectroscopy and magnetic measurements (Figures 9, 11, and 10 12) show that, possibly, the structure is related to the sesquioxide $\mathrm{Er}_{2} \mathrm{O}_{3}$. CD spectroscopy (Figure 10) shows that in all cases, the structure of the peptide is unaffected by silicification, but Raman and CD spectroscopy (Figures 9 and 10) suggest that there may be a change in the peptide structure upon exposure to Er. Finally, 15 two independent cytotoxicity assays (Figures 13 and 14) confirm that the particles exhibit a reasonable biocompatibility towards macrophages derived from human THP-1 cells. The silica-coated, but Er-free particles are less toxic than the uncoated nanoparticles, and the Er-containing particles are less toxic than 20 the particles only coated with silica. This suggest that, over ca. 24 to 48 hours, additional Er in the silica shell exerts a stabilizing effect on the shell, which further reduces the toxicity compared to the Er-free and the completely uncoated particles.

The two key findings of the study are that (1) it is possible to 25 incorporate significant amounts of $\mathrm{Er}$ (which leads to, for example, a specific magnetic signal) into the silica shell and (2) that the nanoparticle toxicology remains essentially unaffected by the addition of Er to the silica shell; the addition Er even seems to reduce the toxicological effects of the resulting particles. The 30 latter point suggests that it should be possible to construct nanoparticles that can be tracked in vivo if, for example, Er is replaced by Eu or another lanthanide, depending on their optical properties. $^{95-98}$

Indeed, Lackowicz ${ }^{99}$ and Deng ${ }^{100}$ have recently reported that 35 the europium quantum yield in silica significantly increases upon inclusion of a metallic silver layer. Moreover, the current study demonstrates that, although the amount of Er incorporated is low, the nanoparticles are clearly visible in SQUID measurements, which could be used for particle detection and tracking.

40 One advantage of our approach over the approaches by Lackowicz $^{99}$ and Deng ${ }^{100}$ is that the tuning of the silica shell is much more straightforward and the polydispersity of the particles is much lower. Moreover, the current work confirms earlier data ${ }^{35}$ that the nanoparticles do not lose their chiral information upon 45 silicification and erbium addition. Essentially, our data show that despite a conformation change of the peptide upon erbium addition (Figures 9 and 10) the CD signal remains the same. This shows that coulombic interaction between the plasmon from the metallic nanoparticle and the covalently linked peptide ${ }^{12}$ may 50 not be the only origin of the observed chiral plasmon resonance. ${ }^{101}$ The CD signal could be also associated with microstructural effects in the particles. ${ }^{12}$

No matter what the exact origin of the chiral information in the CD spectra, chiral nanoparticles are attractive for addressing both

55 fundamental and applied questions. ${ }^{92,102,103}$ Chiral nanoparticles such as the ones presented here can in principle be used in anticounterfeiting or for tracking in a complex matrix such as cells. In the latter case, the Raman spectrum of the nanoparticles can be adapted to not match cellular components. The signal of the 60 particles will then be visible throughout the entire experiment and provide much clearer data than currently available. Finally, our nanoparticles could find application in more complex systems such as new chiral metamaterials and other constructs that are currently investigated for their optical or magnetooptical 65 properties. ${ }^{104-107}$

\section{Conclusion}

Peptide-coated silver nanoparticles are useful, flexible, and adaptable templates and building blocks for multifunctional and biocompatible nanomaterials. Specifically, the incorporation of 70 erbium into the silica coating of a peptide/silver nanoparticle leads to nanoparticles that hold promise for a multitude of applications, in particular in biology and optical materials. The current study has two key findings, namely that (1) it is possible to incorporate measurable amounts of Er into the silica shell on 75 silver nanoparticles and (2) that the cytotoxicity is lower upon Er addition. Furthermore, an APTES layer can be added on the silica surface leading to an increased colloidal stability, a better biocompatibility, and (although this is not discussed here) to further possibilities of chemical and biochemical 80 functionalization. Finally, the chiral information of the hybrid nanoparticles is conserved after silica shell growth and in the presence of erbium. These new modular, multifunctional, bioinspired, and responsive nanoparticles thus constitute an interesting prototype of a whole family of functional 85 nanoparticles with a very large field of application from diagnostics to magnetism and optical devices.

\section{Acknowledgements}

We thank Prof. E.C. Constable for access to his TGA and FTIR spectrometer, the Department of Chemistry (Basel) for access to 90 the CD spectrometer, D. De Bruyn for help with CD measurements, S. Rolf and M. Klimakow for help with XRD and SAXS, and M. Düggelin for help with SEM. A. Mantion thanks the Adolf-Martens e.V. for an Adolf-Martens-Fellowship. A. Mašić is grateful for support by the Alexander von Humboldt ${ }_{95}$ Foundation and the Max Planck Society in the framework of the Max Planck Research Award administered by the Federal Ministry of Education and Research. The Swiss National Science Foundation, University of Potsdam, Fonds der Chemischen Industrie, Bundesanstalt für Materialforschung und -prüfung, 100 Bundesinstitut für Risikobewertung, Université de Strasbourg, Centre Nationale de la Recherche Scientifique, and MPI of Colloids and Interfaces (Colloid Chemistry Department) are thanked for financial support.

\section{References}

105 1. D. D. Evanoff and G. Chumanov, ChemPhysChem, 2005, 6, 12211231.

2. K. S. Lee and M. A. El-Sayed, J. Phys. Chem. B, 2006, 110, 1922019225.

3. X. Chen and H. J. Schluesener, Toxicol. Lett., 2008, 176, 1-12.

110 4. M. Kang, R. Jung, H. S. Kim, J. H. Youk and H. J. Jin, J. Nanosci. Nanotechnol., 2007, 7, 3888-3891. 
5. L. Kvitek, M. Vanickova, A. Panacek, J. Soukupova, M. Dittrich, E. Valentova, R. Prucek, M. Bancirova, D. Milde and R. Zboril, J. Phys. Chem. C, 2009, 113, 4296-4300.

6. A. Henglein and M. Giersig, J. Phys. Chem. B, 1999, 103, 95339539.

7. J. A. Dahl, B. L. S. Maddux and J. E. Hutchison, Chem. Rev. (Washington, DC, U. S.), 2007, 107, 2228-2269.

8. M. C. Daniel and D. Astruc, Chem. Rev. (Washington, DC, U. S.), 2004, 104, 293-346.

10 9. K. J. M. Bishop, C. E. Wilmer, S. Soh and B. A. Grzybowski, Small, 2009, 5, 1600-1630.

10. Y. Min, M. Akbulut, K. Kristiansen, Y. Golan and J. Israelachvili, Nat. Mater., 2008, 7, 527-538.

11. H. Zhang, E. W. Edwards, D. Wang and H. Mohwald, Phys. Chem. Chem. Phys., 2006, 8, 3288-3299.

12. P. Graf, A. Mantion, A. Foelske, A. Shkilnyy, A. Masic, A. F. Thunemann and A. Taubert, Chem.-Eur. J., 2009, 15, 5831-5844.

13. A. Mantion, A. G. Guex, A. Foelske, L. Mirolo, K. M. Fromm, M. Painsi and A. Taubert, Soft Matter, 2008, 4, 606-617.

20 14. A. Mantion, L. Massuger, P. Rabu, C. Palivan, L. B. McCusker and A. Taubert, J. Am. Chem. Soc., 2008, 130, 2517-2526.

15. A. Mantion and A. Taubert, Macromol. Biosci., 2007, 7, 208-217.

16. M. B. Dickerson, K. H. Sandhage and R. R. Naik, Chem. Rev. (Washington, DC, U. S.), 2008, 108, 4935-4978.

25 17. C. Gautier and T. Burgi, J. Am. Chem. Soc., 2006, 128, 11079-11087.

18. C. Gautier and T. Burgi, J. Am. Chem. Soc., 2008, 130, 7077-7084.

19. R. Levy, N. T. K. Thanh, R. C. Doty, I. Hussain, R. J. Nichols, D. J. Schiffrin, M. Brust and D. G. Fernig, J. Am. Chem. Soc., 2004, 126, 10076-10084.

30 20. S. Liu and M.-Y. Han, Chem.-Asian J., 2010, 5, 36-45.

21. L. M. LizMarzan, M. Giersig and P. Mulvaney, Langmuir, 1996, 12, 4329-4335.

22. V. M. Renteria-Tapia, G. Valverde-Aguilar and J. A. Garcia-Macedo, Plasmonics: Metallic Nanostructures and Their Optical Properties V, 2007, 6641, W6411-W6411 (6568).

23. X. F. Sun, C. P. Wei, Q. Y. Li and J. Xu, Chin. J. Inorg. Chem., 2008, 24, 1895-1899.

24. I. Tanahashi, M. Yoshida, Y. Manabe, T. Tohda, S. Sasaki, T. Tokizaki and A. Nakamura, Jpn. J. Appl. Phys., Part 2, 1994, 33,

40 L1410-L1412.

25. M. C. Advincula, P. Patel, P. T. Mather, T. Mattson and A. J. Goldberg, J. Biomed. Mater. Res., Part B, 2009, 88B, 321-331.

26. E. Baeuerlein, Biomineralization, Progress in Biology, Molecular Biology, and Application, Wiley-VCH Verlag GmbH \& Co., Weinheim, Germany, 2004.

27. E. Baeuerlein, Handbook of Biomineralization, Biological Aspects and Structure Formation, Wiley-VCH Verlag GmbH \& Co., Weinheim, Germany, 2007.

28. K. S. Chou and C. C. Chen, Microporous Mesoporous Mater., 2007, 98, 208-213.

29. M. Hildebrand, Chem. Rev. (Washington, DC, U. S.), 2008, 108, 4855-4874.

30. L. Kind, F. A. Plamper, R. Gobel, A. Mantion, A. H. E. Muller, U. Pieles, A. Taubert and W. Meier, Langmuir, 2009, 25, 7109-7115.

55 31. S. Liu, Z. Zhang and M.-Y. Han, Adv. Mater. (Weinheim, Ger.), 2005, 17, 1862-1866.

32. S. V. Patwardhan, R. Maheshwari, N. Mukherjee, K. L. Kiick and S. J. Clarson, Biomacromolecules, 2006, 7, 491-497.

33. M. Sumper and N. Kroger, J. Mater. Chem., 2004, 14, 2059-2065.

60 34. S. Wenzl, R. Hett, P. Richthammer and M. Sumper, Angew. Chem., Int. Ed., 2008, 47, 1729-1732.

35. P. Graf, A. Mantion, A. Haase, A. F. Thunemann, W. Meier, A. Luch and A. Taubert, ACS Nano, 2011, 5, 820-833.

36. M. J. A. de Dood, B. Berkhout, C. M. van Kats, A. Polman and A.

65 van Blaaderen, Chem. Mater., 2002, 14, 2849-2853.

37. F. Enrichi, Ann. N. Y. Acad. Sci., 2008, 1130, 262-266.

38. C. E. Moran, G. D. Hale and N. J. Halas, Langmuir, 2001, 17, 83768379.

39. L. H. Slooff, M. J. A. de Dood, A. van Blaaderen and A. Polman, $70 \quad$ Appl. Phys. Lett., 2000, 76, 3682-3684.
40. V. Sudarsan, S. Sivakumar, F. C. J. M. van Veggel and M. Raudsepp, , Chem. Mater., 2005, 17, 4736-4742.

41. A. C. Marques and R. M. Almeida, J. Non-Cryst. Solids, 2007, 353, 2613-2618.

75 42. J. A. Sampaio and S. Gama, Phys. Rev. B: Condens. Matter Mater. Phys., 2004, 69, 104203

43. V. Matejec, I. Kasik, M. Hayer, D. Berkova, M. Chomat and J. Skokankova, Rev. Roum. Chim., 2002, 47, 1233-1239.

44. N. Daldosso, D. Navarro-Urrios, M. Melchiorri, C. Garcia, P. Pellegrino, B. Garrido, C. Sada, G. Battaglin, F. Gourbilleau, R. Rizk and L. Pavesi, IEEE Journal of Selected Topics in Quantum Electronics, 2006, 12, 1607-1617.

45. D. Navarro-Urrios, Y. Lebour, O. Jambois, B. Garrido, A. Pitanti, N. Daldosso, L. Pavesi, J. Cardin, K. Hijazi, L. Khomenkova, F. Gourbilleau and R. Rizk, J. Appl. Phys., 2009, 106, -.

46. A. Polman and F. C. J. M. van Veggel, J. Opt. Soc. Am. B, 2004, 21, 871-892.

47. C. Strohhofer and A. Polman, Appl. Phys. Lett, 2002, 81, 1414-1416.

48. J. C. Pivin, J. M. de Castro, H. Hofmeister and M. SendovaVassileva, Mater. Sci. Eng., B, 2003, 97, 13-19.

49. A. Bahtat, M. Bouazaoui, M. Bahtat and J. Mugnier, Optics Communications, 1994, 111, 55-60.

50. L. Armelao, S. Gross, G. Obetti and E. Tondello, Surf. Coat. Technol., 2005, 190, 218-222.

95 51. A. J. Kenyon, C. E. Chryssou, C. W. Pitt, T. Shimizu-Iwayama, D. E. Hole, N. Sharma and C. J. Humphreys, J. Appl. Phys., 2002, 91, 367374.

52. B. Dussardier, W. Blanc and G. Monnom, Fiber Integrated Optics, 2008, 27, 484-504.

100 53. M. Mattarelli, M. Montagna, K. Vishnubhatla, A. Chiasera, M. Ferrari and G. C. Righini, Phys. Rev. B: Condens. Matter Mater. Phys., 2007, 75, -.

54. M. Eichelbaum and K. Rademann, Adv. Funct. Mater., 2009, 19, 2045-2052.

105 55. M. A. Zaitoun and S. Al-Tarawneh, J. Lumin., 2011, 131, 1795-1801.

56. M. A. Zaitoun, T. Kim, Q. M. Jaradat, K. Momani, H. A. Qaseer, A. K. El-Qisairi, A. Qudah and N. E. Radwan, J. Lumin., 2008, 128, 227-231.

57. J.-C. G. Bunzli, Chem. Rev. (Washington, DC, U. S.), 2010, 110, 2729-2755.

58. R. C. Leif, L. M. Vallarino, M. C. Becker and S. Yang, Cytometry Part A, 2006, 69A, 767-778.

59. C. Andraud, A. D'Aleo, G. Pompidor, B. Elena, J. Vicat, P. L. Baldeck, L. Toupet, R. Kahn and O. Maury, ChemPhysChem, 2007, $115 \quad 8,2125-2132$.

60. S. R. Kline, J. Appl. Crystallogr., 2006, 39, 895-900.

61. P. Bartlett and R. H. Ottewill, J. Chem. Phys., 1992, 96, 3306-3318.

62. G. V. Schulz, Z. Phys. Chem. (Muenchen, Ger.), 1935, 30, 379-398.

63. M. Kotlarchyk and S. H. Chen, J. Chem. Phys., 1983, 79, 2461-2469.

120 64. J. BrunnerPopela and O. Glatter, J. Appl. Crystallogr., 1997, 30, 431442.

65. R. Mittelbach and O. Glatter, J. Appl. Crystallogr., 1998, 31, 600608.

66. J. A. Potton, G. J. Daniell and B. D. Rainford, J. Appl. Crystallogr., 1988, 21, 663-668.

67. J. Ilavsky and P. R. Jemian, J. Appl. Crystallogr., 2009, 42, 347-353.

68. O. Paris, C. H. Li, S. Siegel, G. Weseloh, F. Emmerling, H. Riesemeier, A. Erko and P. Fratzl, J. Appl. Crystallogr., 2007, 40, S466-S470.

130 69. A. P. Hammersley, S. O. Svensson, M. Hanfland, A. N. Fitch and D. Hausermann, High Pressure Research, 1996, 14, 235 - 248.

70. J. Rodríguez-Carvajal, Physica B+C 1993, 192, 55-69.

71. G. Bohm, R. Muhr and R. Jaenicke, Protein Eng., 1992, 5, 191-195.

72. J. Auwerx, Experientia, 1991, 47, 22-31.

135 73. E. K. Park, H. S. Jung, H. I. Yang, M. C. Yoo, C. Kim and K. S. Kim, Inflamm Res, 2007, 56, 45-50.

74. B. Wu, M. Zinkevich, F. Aldinger, D. Wen and L. Chen, J. Solid State Chem., 2007, 180, 3280-3287.

75. A. Fert, Bulletin de la Societe Francaise de Mineralogie et de 140 Cristallographie, 1962, 85, 267-270. 
76. G. Adachi and N. Imanaka, Chem. Rev. (Washington, DC, U. S.), 1998, 98, 1479-1514.

77. K. Larson-Smith, A. Jackson and D. C. Pozzo, J. Colloid Interface Sci., 2010, 343, 36-41.

5 78. J. J. Mock, M. Barbic, D. R. Smith, D. A. Schultz and S. Schultz, J. Chem. Phys., 2002, 116, 6755-6759.

79. I. O. Sosa, C. Noguez and R. G. Barrera, J. Phys. Chem. B, 2003, 107, 6269-6275.

80. S. Mukherjee, C. H. Chen, C. C. Chou, K. F. Tseng, B. K. Chaudhuri and H. D. Yang, Phys. Rev. B: Condens. Matter, 2010, 82, 104107.

81. E. Staritzky, Anal. Chem., 1956, 28, 2023-2024.

82. A. M. Lejus and D. Michel, Phys. Status Solidi B, 1977, 84, K105K108.

83. C. Benelli and D. Gatteschi, Chem. Rev. (Washington, DC, U. S.), $15 \quad 2002,102,2369-2387$.

84. A. Okazawa, R. Watanabe, H. Nojiri, T. Nogami and T. Ishida, Polyhedron, 2009, 28, 1808-1813.

85. A. Maqsood, B. M. Wanklyn and G. Garton, J. Cryst. Growth, 1979, 46, 671-680.

20 86. M. J. M. Leask, P. R. Tapster and M. R. Wells, J. Phys. C Solid State Phys, 1986, 19, 1173-1187.

87. A. Maqsood, J. Mater. Sci., 1981, 16, 2198-2204.

88. R. M. Moon, W. C. Koehler, H. R. Child and L. J. Raubenhe, Phys. Rev. Lett., 1968, 1, 722-731.

25 89. E. F. Bertaut and R. Chevalier, C. R. Acad. Sci., Paris, Ser. B, 1966, 262B, 1707-1710.

90. J. Blanusa, B. Antic, A. Kremenovic, A. S. Nikolic, L. Mazzerolles, S. Mentus and V. Spasojevic, Solid State Commun., 2007, 144, 310314.

30 91. S. Mukherjee, P. Dasgupta and P. K. Jana J. Phys. D: Appl. Phys., 2008, 41, 215004.

92. C. Noguez and I. L. Garzon, Chem. Soc. Rev., 2009, 38, 757-771.

93. A. Haase, H. F. Arlinghaus, J. Tentschert, H. Jungnickel, P. Graf, A. Mantion, F. Draude, S. Galla, J. Plendl, M. E. Goetz, A. Masic, W.

35 Meier, A. F. Thunemann, A. Taubert and A. Luch, ACS Nano, 2011, 5, 3059-3068.

94. A. Haase, J. Tentschert, H. Jungnickel, P. Graf, A. Mantion, F. Draude, J. Plendl, M. E. Goetz, S. Galla, A. Mašić, A. F. Thunemann, A. Taubert, H. F. Arlinghaus and A. Luch, Journal of Physics: Conference Series, 2011, 304, 012030.

95. E. G. Moore, J. D. Xu, C. J. Jocher, E. J. Werner and K. N. Raymond, J. Am. Chem. Soc., 2006, 128, 10648-10649.

96. T. Nishioka, J. L. Yuan, Y. Yamamoto, K. Sumitomo, Z. Wang, K. Hashino, C. Hosoya, K. Ikawa, G. L. Wang and K. Matsumoto, 45 Inorg. Chem., 2006, 45, 4088-4096.

97. H. Takalo, V. M. Mukkala, L. Merio, J. C. RodriguezUbis, R. Sedano, O. Juanes and E. Brunet, Helv. Chim. Acta, 1997, 80, 372387.

98. H. Takalo, V. M. Mukkala, H. Mikola, P. Liitti and I. Hemmila, Bioconjugate Chem., 1994, 5, 278-282.

99. J. Zhang, Y. Fu and J. R. Lakowicz, J. Phys. Chem. C, 2009, 113, 19404-19410.

100.W. Deng, D. Y. Jin, K. Drozdowicz-Tomsia, J. L. Yuan and E. M. Goldys, Langmuir, 2010, 26, 10036-10043.

55 101.A. O. Govorov, Z. Y. Fan, P. Hernandez, J. M. Slocik and R. R. Naik, Nano Lett., 2010, 10, 1374-1382.

102.C. Gautier and T. Burgi, ChemPhysChem, 2009, 10, 483-492.

103.V. I. Sokolov, Russ. J. Coord. Chem., 2009, 35, 553-565.

104.D. Chakravorty, S. Basu, P. K. Mukherjee, S. K. Saha, B. N. Pal, A. Dan and S. Bhattacharya, J. Non-Cryst. Solids, 2006, 352, 601-609.

105.D. Chakravorty, S. Basu, B. N. Pal, P. K. Mukherjee, B. Ghosh, K. Chatterjee, A. Bose, S. Bhattacharya and A. Banerjee, Bull. Mater. Sci., 2008, 31, 263-276.

106.H. B. Lee, Y. M. Yoo and Y. H. Han, Scr. Mater., 2006, 55, 1127651129.

107.W. J. Li and T. Sun, Mater. Chem. Phys., 2009, 116, 164-168. 